\title{
Further Steps towards Driver Modeling According to the Bayesian Programming Approach
}

\author{
Claus Möbus and Mark Eilers* \\ University of Oldenburg / OFFIS, Germany \\ \{claus.moebus, mark.eilers\}@uni-oldenburg.de
}

\begin{abstract}
The Human Centered Design (HCD) of Partial Autonomous Driver Assistance Systems (PADAS) requires Digital Human Models (DHMs) of human control strategies for simulating traffic scenarios. We describe first results to model lateral and longitudinal control behavior of drivers with simple $d y$ namic Bayesian sensory-motor models according to the Bayesian Programming (BP) approach: Bayesian Autonomous Driver (BAD) models. BAD models are learnt from multivariate time series of driving episodes generated by single or groups of users. The variables of the time series describe phenomena and processes of perception, cognition, and action control of drivers. BAD models reconstruct the joint probability distribution (JPD) of those variables by a composition of conditional probability distributions (CPDs). The real-time control of virtual vehicles is achieved by inferring the appropriate actions under the evidence of sensory percepts with the help of the reconstructed JPD.
\end{abstract}

Keywords: digital human response models, driver models, Bayesian autonomous driver models, learning of human control strategies, probabilistic Bayesian lateral and longitudinal control, graphical modeling, human behavior learning and transfer, Bayesian Programming.

\section{Introduction}

A driver is a human agent whose skills can be described by stages which were labeled by Anderson [1] as: cognitive, associative, and autonomous. According to these stages various modeling approaches seem to be adequate: production-system (e.g. models in the ACT-R-architecture $[2,3]$ ) for the cognitive and associative stages, control-theoretic [4 - 6], and probabilistic models [7, 8] for the autonomous stage. The first two kinds of models are quite standard approaches, now [9]. The main advantage of the new probabilistic models is that they have a clean semantics and at the same time are more robust than the other approaches $[10,11]$. This is a great achievement in consequence of the irreducible inter- and intra-individual variability of human behavior and the irreducible incompleteness of knowledge about the stochastic environment, the driver, and his psychological mechanisms [12, 13]. Furthermore, probabilistic computational models are not programmed like traditional simulation

\footnotetext{
* Project Integrated Modeling for Safe Transportation (IMOST) sponsored by the Government of Lower Saxony, Germany under contracts ZN2245, ZN2253, ZN2366.
} 
software but are condensed and abstracted in an objective manner. In this way the behavior of single or groups of drivers can be learnt by objective machine learning techniques [14].

The intra-individual variation of human behavior can e.g. be seen from the gaze distribution of a single driver passing the same road position repeatedly (Fig. $1^{1}$ ).

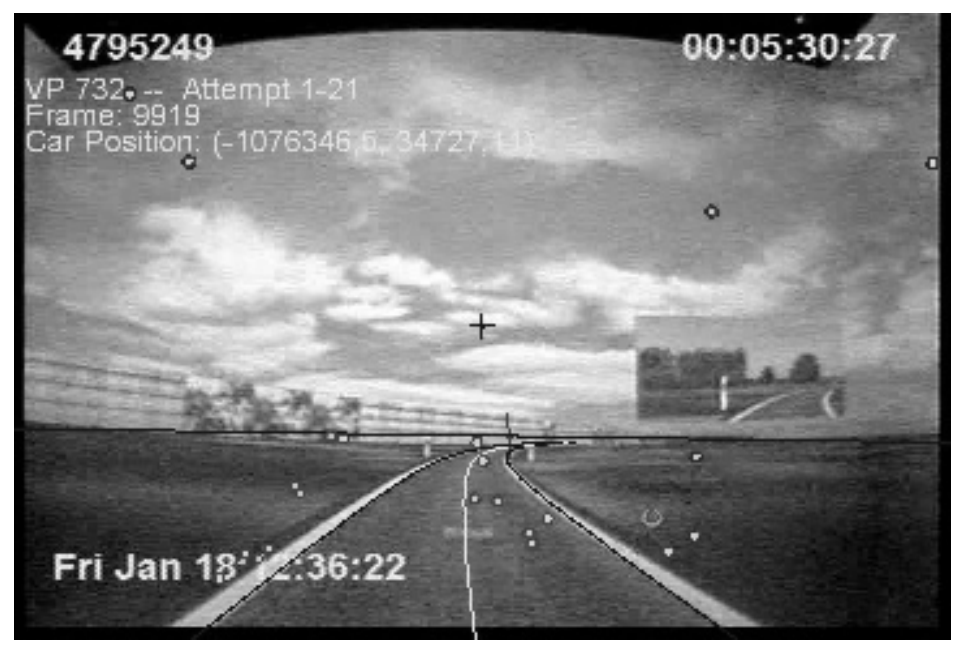

Fig. 1. Distribution of the drivers gaze on a single lane road; gazes of 21 drives of one driver within a radius of $50 \mathrm{~cm}$ around the current position are shown ${ }^{2}$

Gazes are directed to various positions in the vision field, which conflicts with assumptions made in control-theoretic driver models like Salvucci \& Gray's 2-pointmodel of lateral control $[2,15]$.

In summary computational driver models should

- predict and generate driver behavior emitted by drivers sometimes in interaction with assistance systems

- identify situations or maneuvers and classify behavior of drivers as e.g. anomalous or normal

- provide a robust and valid mapping from human sensory data to human control actions even when inter- and intra-individual variance is observable

\footnotetext{
${ }^{1}$ Background image taken from experimental records at DLR, Braunschweig, Germany. Calculations of the gaze points have been done at OFFIS by Bertram Wortelen.

${ }^{2}$ During the experiments at the DLR data from the simulator and the eye tracking system have been recorded at a frequency of $30 \mathrm{~Hz}$. The eye tracking data together with the position of the car on the road and a geometrical representation of the road have been used to calculate the drivers' focal gaze points on the road and to draw this as an overlay on the video. This figure contains the focal gaze points of all 21 drives of one driver at the same road position. The course of the cars deviates in each run, so actually we have taken all focal points within a radius of $50 \mathrm{~cm}$. These points are drawn as red dots in the figure.
} 
- be learnt from time series of raw data or empirical probability distributions with statistical sound (machine-learning) procedures relying only on a few non-testable ad hoc or axiomatic assumptions

- be able to learn new patterns of behavior without forgetting already learnt skills (stability-plasticity dilemma) [16].

\section{Bayesian Autonomous Driver (BAD) Models}

\subsection{Related Work}

Due to the irreducible variability of human behavior and the irreducible lack of knowledge about cognitive mechanisms of a driver in a stochastic environment it seems rational to model human drivers with for instance probabilistic models: Bayesian Autonomous Driver (BAD) models. According to the Bayesian Programming (BP) approach $[13,17,23]$ BAD models $[7,8]$ are a special type of Bayesian Networks (BN) [18 - 22] using concepts from probabilistic robotics [11].

BP is a simple and generic framework suitable for human modeling in the presence of incompleteness and uncertainty. It provides integrated model-driven data analysis and model construction. In contrast to conventional Bayesian networks BP-models may have a recursive structure and infer concrete motor actions for real-time control on the basis of sensory evidence. Actions are sampled from CPDs for action variables after propagating sensor or task goal evidence. Sampling can be made with the draw or the best operator. Sampling with draw is sampling of concrete actions from the CPD P(Action I parents ${ }^{3}$ (Action)) and sampling with best is sampling the conditional expected value of the CPD P(Action I parents(Action)). This is known as the nonlinear regression of Action on parents(Action): E(Action I parents(Action)).

BAD models can be learnt objectively with statistical sound methods from multivariate time series of the variables of interest. They describe phenomena on the basis of these variables and the decomposition of their JPD by (simplified) CPDs according to the special chain rule for Bayesian networks [22, p.36]. The underlying (conditional) independence hypotheses (CIHs) between sets of variables can be tested by standard statistical methods (e.g. the conditional mutual information index [22, p.237]). Model validity is thus included in the modeling process by model-driven data-analysis without ex-post validation.

In [7] we described first steps to model lateral and longitudinal control behavior of single and groups of drivers with reactive Bayesian sensory-motor models. Here we include the time domain and describe work in progress with dynamic Bayesian sensory-motor models. The vision is a dynamic BAD model which is able to compose behavior from basic kinds of motor schemas: dynamic mixture-of-expert (MoE; Fig. 9) [23] BAD model [8]. This new MoE models facilitate the collection of sensorymotor schemas (= experts) in a library. Context dependent driver behavior could be generated by mixing pure behavior from different schemas (= experts) avoiding the stability-plasticity dilemma [16] at the same time. Whereas [24] rely on Hidden Markov Models (HMMs) for learning fine manipulation tasks like grasping and

\footnotetext{
${ }^{3}$ parents(Action) are the parents of the node Action in the $\mathrm{BN}[22, \mathrm{p} .36]$.
} 
assembly by Markov mixtures of experts we strive for a new dynamic Bayesian Network (DBN) model in learning multi-maneuver driving behavior [8].

\subsection{Basic Concepts}

In presenting basic concepts of BP like postulates, definitions, notations and rules we borrow from $[13,17]$. A BP is defined as a mean of specifying a family of probability distributions. By using such a specification it is possible to construct a BAD model, which can effectively control a (virtual) vehicle. The components of a BP are presented in Fig. 2, and Fig. 3, where the analogy to a logic program is helpful.

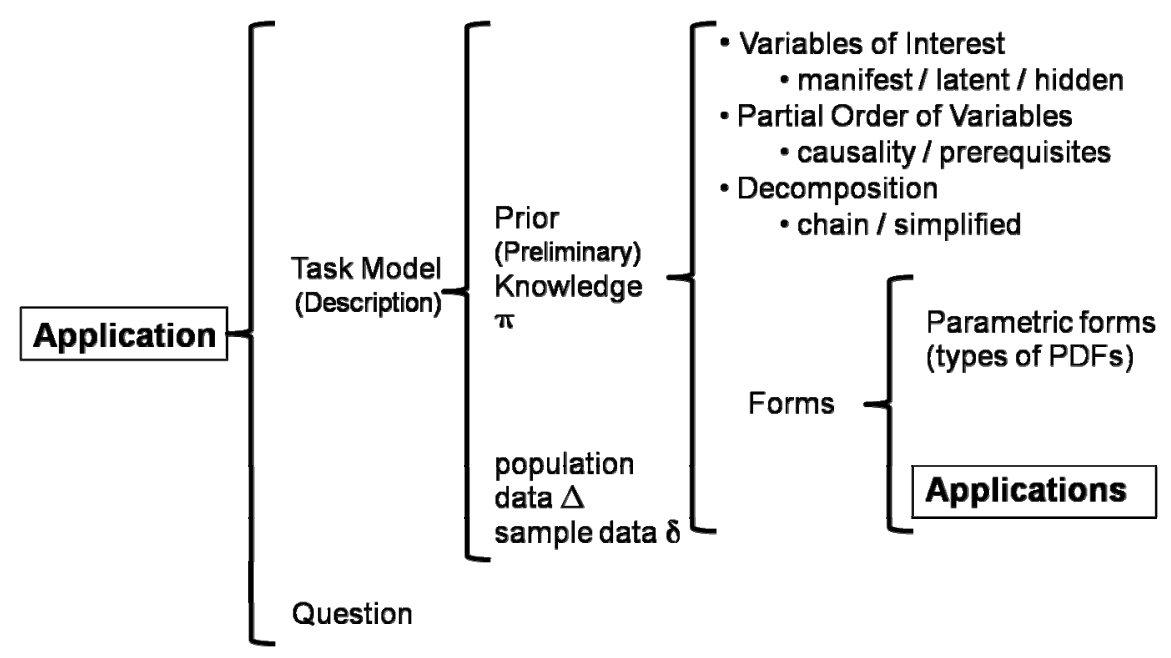

Fig. 2. Structure of a Bayesian Program (slightly modified terminology to [13, 17])

An application consists of a (Task model) description and a question. A description is constructed from preliminary knowledge and a data set. Preliminary knowl$e d g e$ is constructed from a set of pertinent variables, a decomposition of the JPD and a set of forms. Forms are either parametric forms or BPs.

The purpose of a description is to specify an effective method to compute a JPD on a set of variables given a set of (experimental) data and preliminary knowledge. To specify preliminary knowledge the modeler must define the set of relevant variables on which the JPD is defined, decompose the JPD into factors of CPDs according to CIHs, and define the forms. Each CPD in the decomposition is a form. Either this is a parametric form which parameter are estimated from data or another application. Given a description a question is obtained by partitioning the variables into searched, known, and unknown variables. We define a question as the CPD P(Searched I Known, preliminary knowledge, data). The selection of an appropriate action can be treated as the inference problem: Policy( $\mathrm{P}($ Action I Percepts, Goals, preliminary knowledge, data). Various policies (Draw, Best, and Expectation) are possible whether the concrete action is drawn at random, chosen as the best action with highest probability, or as the expected action. 


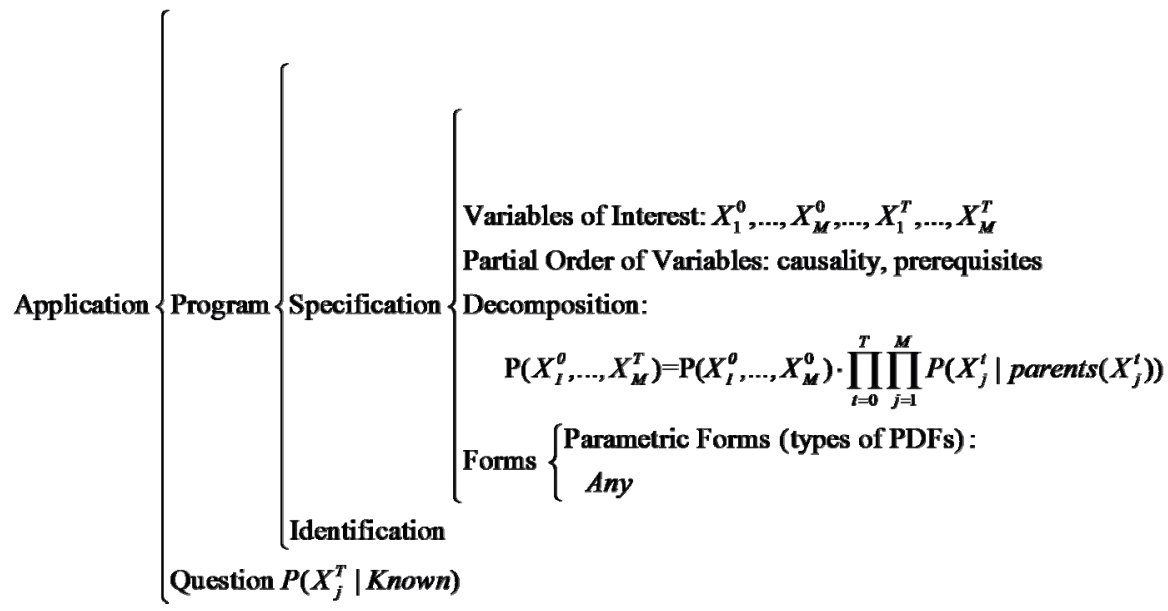

Fig. 3. Structure of Dynamic Bayesian Networks (DBNs) as Bayesian Programs (slightly modified terminology to [23])

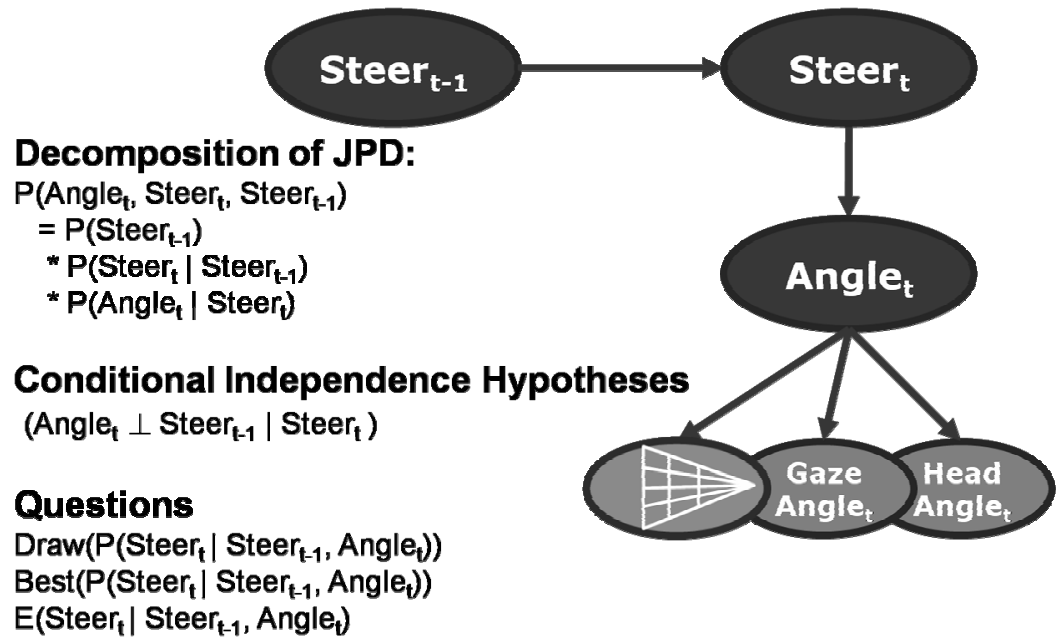

Fig. 4. Partially inverse DBN of Lateral Control

\subsection{Results}

Static reactive or static inverse models have not been satisfactory because they generate behavior which is more erratic and nervous than human behavior [7]. Better results can be obtained by introducing a memory component and using DBNs. In a first step we estimated two DBNs separately for the lateral and longitudinal control. Our experience is that partially inverse models were quite useful (Fig. 4, 5).

In an inverse model arcs in the DAG of the graphical model are directed from the consequence to the prerequisites. The semantics of these arcs are denoted by the 


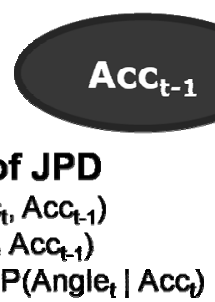

Decomposition of JPD

$P\left(\right.$ Speed $_{t,}$, Angle $_{t}$, Acc $_{1}$, Acc $\left._{t-1}\right)$

$=P\left(A c c_{-1}\right) * P\left(A c c_{1} \mid A c c_{1-1}\right)$

${ }^{*} P\left(\right.$ Speed $_{t} \mid$ Acc $){ }^{*} P\left(\right.$ Angle $_{t} \mid$ Acc $\left._{t}\right)$

Conditional Independence Hypotheses

(Speed $_{\mathrm{t}} \perp\left\{\right.$ Angle $_{\mathrm{t}}$, AcG $\left._{\mathrm{i}-1}\right\} \mid$ AcG $_{\mathrm{t}}$ )

(Angle ${ }_{t} \perp$ AcG $_{t-1} \mid A c C_{t}$ )

\section{Questions}

$\operatorname{Draw}\left(P\left(A c c_{1} \mid A_{c c_{1-1}}\right.\right.$, Angle, Speed $\left.)\right)$

$\operatorname{Best}\left(P\left(\right.\right.$ Acc $_{t} \mid$ Acc $_{t-1}$, Angle $_{t}$, Speed $\left.\left.t\right)\right)$

$E\left(P\left(\right.\right.$ AcG $_{t} \mid$ AcG $_{t-1}$, Angle $_{t}$, Speed $\left.\left._{t}\right)\right)$

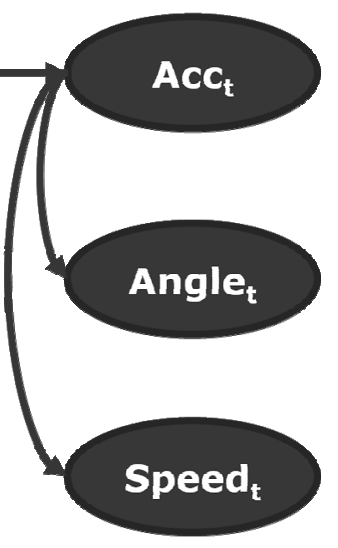

Fig. 5. Partially inverse DBN of Longitudinal Control

conditional probabilities $\mathrm{P}$ (Prerequisites | Consequence). Our models are partially inverse because because most arcs are inverted but the arcs between time slice $\mathrm{t}-1$ and $\mathrm{t}$ are in causal order from prerequisites to consequences. The variables of interest are partitioned into sensory variables (heading angle of the vehicle, perceived speed) and actions (steering angle, acceleration). According to the visual attention allocation theory of Horrey et al. [25] the perception of the heading angle is influenced by areas in the visual field (ambient channel), the head angle and the gaze angle relative to the head. At the present moment light grey nodes in Fig. 4 are not included into the

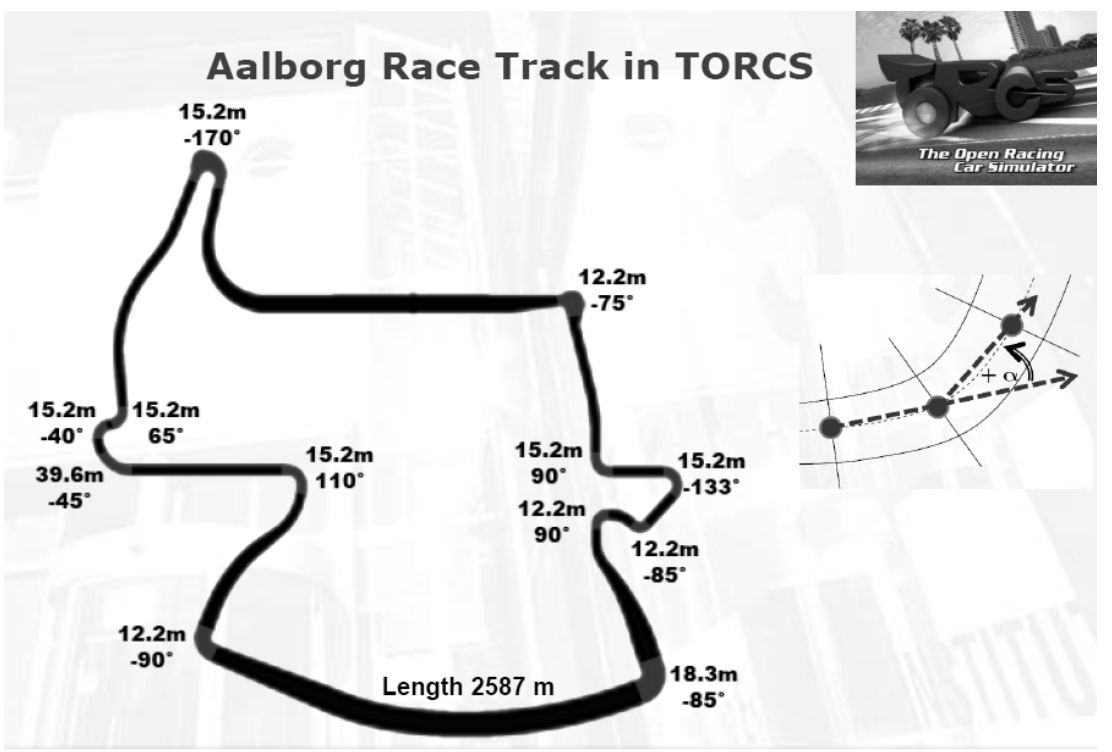

Fig. 6. Bird's eye view of race track with curve radii and rotation angles 


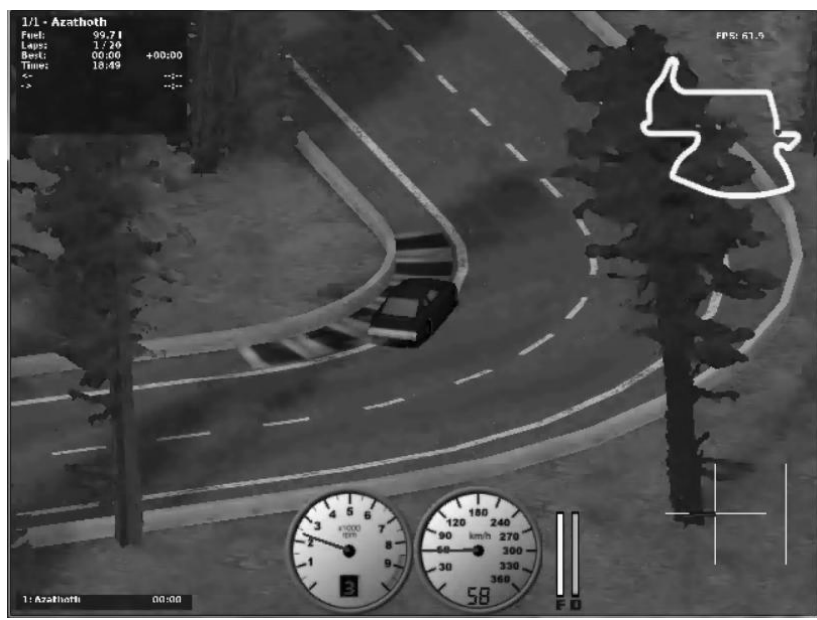

Fig. 7. Snapshot of BAD model drive on TORCS race track

model. Instead we assumed that drivers are able to compute the aggregate sensory variables heading angle and vehicle speed. Compared to the lateral control in Salvucci \& Gray's model [2] our BAD model is simpler and makes less assumptions about the vision field.

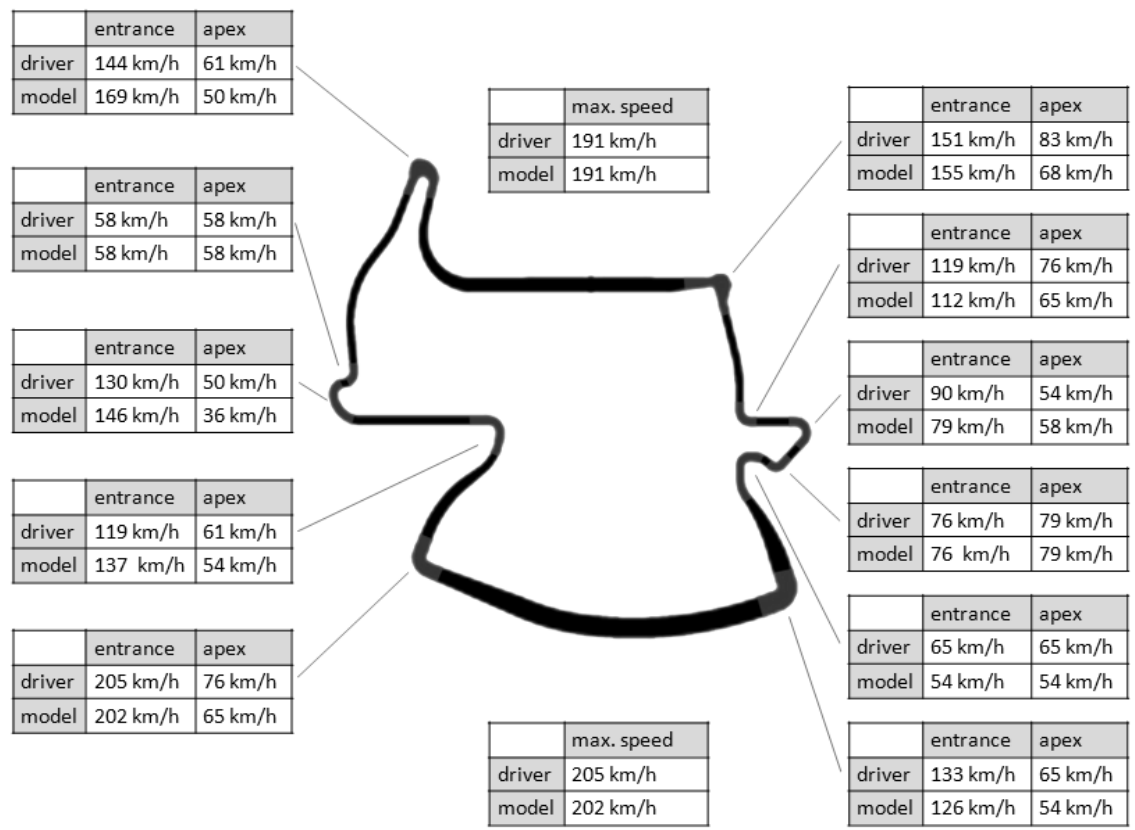

Fig. 8. Comparison of Human and BAD-Model Drives 


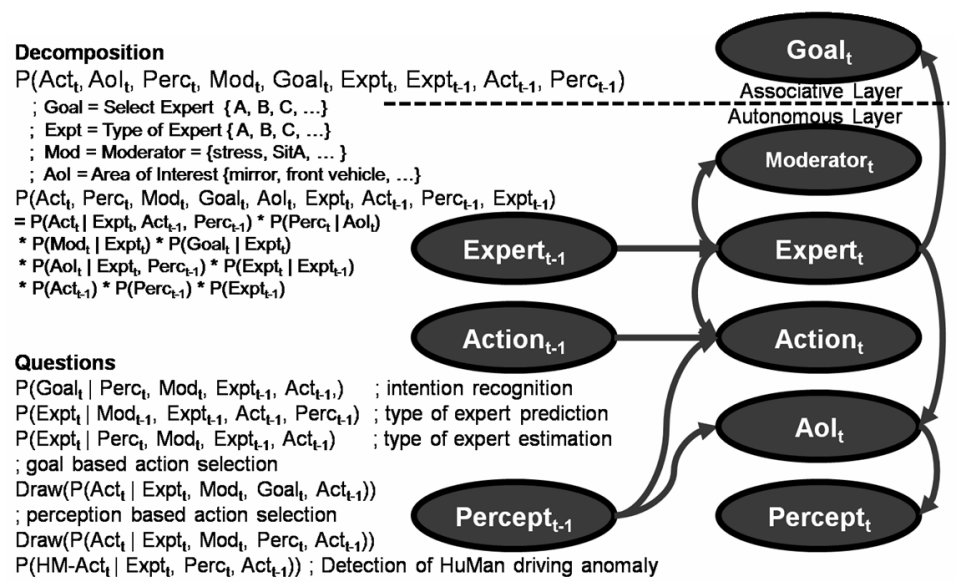

Fig. 9. Mixture-of-Experts Architecture of Bayesian Autonomous Driver (BAD) Model

Data were obtained in experimental drives of a single driver on the TORCS racing course (Fig. 6, [26]). Only a few laps were necessary to obtain the data for estimating the parameters (means, standard deviations) for the Gaussian parametric forms. A snapshot of a BAD model drive is shown in Fig. 7.

The map of the racing course and curve specific measurements is presented in Fig.6, 8. A comparison of the driver speed data with model generated speed data demonstrates the quality of the simple BAD model. But, because there are some collisions with the roadsides the capabilities of the BAD model need to be improved.

Further improvements are expected by combining the two controllers, by including cognitive constructs like goals and latent states of the driver, and above all segmenting behaviors into context dependent schemas (= experts) (Fig. 9).

Using goals (e.g. driving a hairpin or an S-curve) makes it possible to adapt the model to different road segments and situations. We expect to use the same model for situation recognition or to situation-adapted control. The modeling idea of a HMM was abandoned because the state variable has to very fine grained to obtain a high quality vehicle control [8]. In HMMS perceptions and actions should be conditionally independent, when state is known.

\section{Conclusions and Outlook}

In our current research [7, 8] we strive for the realization of BAD model architecture on the basis of a DBN (Fig. 9). It is a psychological motivated mixture-of-experts model which is distributed across two time slices. It implements the autonomous layer of a cognitive agent and avoids the fine grained state assumptions of HMMs. Learning data are time series of pertinent variables: percepts, goals, and actions. We can model individual or groups of human and artificial agents. The model propagates information in various directions. When working top-down, goals emitted by the associative layer of a cognitive model a corresponding expert, who propagates actions, areas of interest (AoIs) and perceptions is selected. When working bottom-up, percepts trigger AoIs, actions, experts and goals. When the task or goal is defined and 
the model has certain percepts evidence can be propagated simultaneously top-down and bottom-up and the appropriate expert and its behavior can be activated. Thus, the model can be easily extended to implement the SEEV visual scanning model [25].

All probabilistic models presented here can be constructed by data mining single or aggregated driver's behavior traces in experimental settings with or without experimental induced goals.

\section{References}

1. Anderson, J.R.: Learning and Memory. John Wiley, Chichester (2002)

2. Salvucci, D.D., Gray, R.: A Two-Point Visual Control Model of Steering. Perception 33, 1233-1248 (2004)

3. Salvucci, D.D.: Integrated Models of Driver Behavior. In: Gray, W.D. (ed.) Integrated models of cognitive systems, pp. 356-367. Oxford University Press, New York (2007)

4. Jürgensohn, T.: Control Theory Models of the Driver. In: Cacciabue (ed.), pp. 277-292 (2007)

5. Weir, D.H., Chao, K.C.: Review of Control Theory Models for Directional and Speed Control. In: Cacciabue, P.C., pp. 293-311 (2007)

6. Möbus, C., Hübner, S., Garbe, H.: Driver Modelling: Two-Point-or Inverted Gaze-BeamSteering. In: Rötting, M., Wozny, G., Klostermann, A., Huss, J. (Hrsgb) Prospektive Gestaltung von Mensch-Technik-Interaktion, 7. Berliner Werkstatt Mensch-MaschineSysteme, Berlin, Fortschritt-Berichte VDI-Reihe 22 (Nr. 25), pp. 483-488. VDI Verlag, Düsseldorf (2007)

7. Möbus, C., Eilers, M.: First Steps Towards Driver Modeling According to the Bayesian Programming Approach, Symposium Cognitive Modeling. In: Urbas, L., Goschke, T., Velichkovsky, B. (eds.) KogWis, vol. 6, p. 59. Christoph Hille, Dresden (2008)

8. Möbus, C., Eilers, M., Garbe, H., Zilinski, M.: Probabilistic, and Empirical Grounded Modeling of Agents in Partial Cooperative (Traffic) Scenarios. In: Conference Proceedings, HCI 2009, Digital Human Modeling. LNCS (LNAI). Springer, San Diego (2009)

9. Cacciabue, P.C. (ed.): Modelling Driver Behaviour in Automotive Environments. Springer, London (2007)

10. Chater, N., Oxford, M. (eds.): The Probabilistic Mind: Prospects for Bayesian Cognitive Science. Oxford University Press, Oxford (2008)

11. Thrun, S., Burgard, W., Fox, D.: Probabilistic Robotics. MIT Press, Cambridge (2005)

12. Anderson, J.R., Fincham, J.M., Qin, Y., Stocco, A.: A Central circuit of the mind. Trends in Cognitive Science 12(4), 136-143 (2008)

13. Bessiere, P., Laugier, C., Siegwart, R. (eds.): Probabilistic Reasoning and Decision Making in Sensory-Motor Systems. Springer, Berlin (2008)

14. Xu, Y., Lee, K.K.C.: Human Behavior Learning and Transfer. CRC Press, Boca Raton (2006)

15. Möbus, C., Hübner, S., Garbe, H.: Driver Modelling: Two-Point- or Inverted Gaze-BeamSteering. In: Rötting, M., Wozny, G., Klostermann, A., Huss, J. (eds.) Prospektive Gestaltung von Mensch-Technik-Interaktion, Fortschritt-Berichte VDI-Reihe 22 (Nr. 25), pp. 483-488. VDI Verlag, Düsseldorf (2007)

16. Hamker, F.H.: RBF learning in a non-stationary environment: the stability-plasticity dilemma. In: Howlett, R.J., Jain, L.C. (eds.) Radial Basis Function networks 1: Recent Developments in Theory and Applications; Studies in fuzziness and soft computing, ch. 9, vol. 66, pp. 219-251. Physica Verlag, Heidelberg (2001) 
17. Lebeltel, O., Bessiere, P., Diard, J., Mazer, E.: Bayesian Robot Programming. Autonomous Robots 16, 49-79 (2004)

18. Pearl, J.: Probabilistic Reasoning in Intelligent Systems. Morgan Kaufmann, San Mateo (1988)

19. Pearl, J.: Causality: Models, Reasoning and Interference, 2nd edn. Cambridge University Press, Cambridge (2009)

20. Spirtes, P., Glymour, C., Scheines, R.: Causation, Prediction, and Search, 2nd edn. MIT Press, Cambridge (2001)

21. Neapolitan, R.E.: Learning Bayesian Networks. Prentice Hall, Upper Saddle River (2004)

22. Jensen, F.V., Nielsen, T.D.: Bayesian Networks and Decision Graphs, 2nd edn. Springer, Heidelberg (2007)

23. Bessiere, P.: Survey: Probabilistic Methodology and Techniques for Artifact Conception and Development, Repport de Recherche, No. 4730, INRIA (2003)

24. Meila, M., Jordan, M.I.: Learning Fine Motion by Markov Mixtures of Experts, MIT, AI Memo No. 1567 (1995)

25. Horrey, W.J., Wickens, C.D., Consalus, K.P.: Modeling Driver's Visual Attention Allocation While Interacting With In-Vehicle Technologies. J. Exp. Psych. 12, 67-78 (2006)

26. TORCS, http://torcs. sourceforge. net/ (visited 18.10, 2008) 